\title{
Locally-advanced non-small cell lung cancer: shall immunotherapy be a new chance?
}

\author{
Andrea Riccardo Filippi ${ }^{1}$, Jacopo Di Muzio ${ }^{1}$, Serena Badellino ${ }^{2}$, Cristina Mantovani ${ }^{2}$, Umberto Ricardi ${ }^{1}$ \\ ${ }^{1}$ Department of Oncology, University of Torino, Torino, Italy; ${ }^{2}$ Radiation Oncology Department, Città della Salute e della Scienza University \\ Hospital, Torino, Italy \\ Contributions: (I) Conception and design: AR Filippi, U Ricardi; (II) Administrative support: None; (III) Provision of study materials or patients: J Di \\ Muzio, S Badellino, C Mantovani; (IV) Collection and assembly of data: J Di Muzio, S Badellino; (V) Data analysis and interpretation: AR Filippi, J \\ Di Muzio, S Badellino; (VI) Manuscript writing: All authors; (VII) Final approval of manuscript: All authors. \\ Correspondence to: Andrea Riccardo Filippi, MD. Department of Oncology, University of Torino, Regione Gonzole 10, 10043 Orbassano (Torino), \\ Italy. Email: andreariccardo.filippi@unito.it.
}

\begin{abstract}
Locally advanced non-small cell lung cancer (NSCLC) represents approximately one third of presentations at diagnosis. Most patients are judged non-surgical due to disease extension, and chemoradiotherapy still represents the standard therapeutic option, with unsatisfactory results in terms of overall survival (OS) despite advances in staging and radiation therapy planning and delivery. Immunotherapy, and in particular immune-checkpoint inhibitors targeting the PD-1/PD-L1 axis, gained wide popularity for NSCLC in light of the positive findings of several trials in metastatic disease. Stage III unresectable NSCLC is a remarkably interesting setting for the combined use of chemo-radiation and immunotherapy, also considering the multiple experimental evidences in favor of a synergistic effect between radiation and immune checkpoint inhibitors, with the potential of enhancing immuno-modulating effects and overcoming resistance. We here summarized the biological rationale and the initial clinical experiences testing for this combination, and we briefly discussed ongoing trials and future options in this field.
\end{abstract}

Keywords: Radiotherapy; immunotherapy; locally advanced non-small cell lung cancer (NSCLC)

Submitted Dec 04, 2017. Accepted for publication Dec 06, 2017.

doi: $10.21037 /$ jtd.2017.12.53

View this article at: http://dx.doi.org/10.21037/jtd.2017.12.53

\section{Introduction}

According to the tumor node metastases (TNM) international staging system, approximately thirty percent of patients affected with non-small cell lung cancer (NSCLC) are diagnosed with "locally advanced" disease (1). This group includes a wide spectrum of clinical presentations with often a considerable tumor burden (T3-T4 and N2-N3). Beyond stages III A and B, the latest TNM ( $8^{\text {th }}$ edition) also introduces stage IIIC, which refers to a massive parenchymal localization combined with contralateral lymph nodes involvement (T3-T4 and N3) (2). Most of stage III NSCLC patients are judged as nonsurgical given the disease extension. Despite the absence of metastases their prognosis is severe (with differences across sub-stages), with 5-year overall survival (OS) rate of approximately $20 \%$ after concomitant or sequential chemo-radiation (3). As in phase 2 trials a progressive increase in radiation dose resulted associated to better localregional control and OS, Radiation Therapy Oncology Group (RTOG) conducted a prospective phase 3 study to establish the safety and efficacy of increasing total radiation dose with concurrent carboplatin-paclitaxel, comparing 60 vs. 74 Gy, with negative results (RTOG0617) (4). The control arm (60 Gy) of this study reached a median OS of 28.7 months, a result previously unseen in any historical study, now representing a new benchmark for comparison. On the front of radiation dose escalation, other groups tested the possibility of delivering a stereotactic boost after conventional radiation therapy (RT) in order to selectively 
increase tumor dose, with promising results in terms of feasibility and local control without survival benefits, and still unconfirmed results by high-quality prospective studies $(5,6)$. Although the outcomes after concomitant chemoradiation in this setting appear better than before, still not all patients are eligible, making this therapeutic choice limited to a selected group of generally younger and fit patients. Beyond radiation dose escalation, a logical step for improving survival for non-surgical stage III patients was to investigate for the combination of chemo-radiotherapy with targeted agents (gefitinib, erlotinib) and/or antiangiogenic therapies (bevacizumab), following the positive results obtained for these drugs in stage IV. However, these attempts did fail in increasing survival, while adding significant toxicity (7).

A new window of opportunity emerged when immunotherapy gained exceptional popularity for its high efficacy for a heterogeneous group of metastatic solid tumors, including NSCLC. The first clinical applications, in particular the use of immune checkpoint inhibitors targeting the PD-1/PD-L1 axis, produced remarkable results in metastatic NSCLC, especially in patients overexpressing PD-L1 on cancer cells (8-11). These innovative immunemodulators were then tested for locally advanced disease in pivotal trials, and we here summarize the results obtained so far, as well as the ongoing trials and our perspective on the future options in this setting.

\section{Rationale for the combination of immunotherapy and radiotherapy in NSCLC}

Radiotherapy has consistently been shown to activate key elements of the immune system that may be responsible for resistance to immunotherapy (12-16); at the same time, radio(chemo)therapy may synergize with immunotherapy and possibly overcome resistance and potentiate the pro-immunogenic effects $(17,18)$. As shown by several experimental studies, RT may convert a poorly immunogenic tumor into an immunogenic one, by increasing antigen release, T-cells priming and crosspriming in lymph nodes, T-cells trafficking to tumor site, and increased expression of MHC-class 1 molecules (19-21). These effects are partially counterbalanced by immunosuppressive effects, one of the most important being the enhanced PD-L1 expression on cancer cells, that can be neutralized promoting a synergistic effect when anti-PD-L1 agents are used in combination with RT (22). Targeting the PD-1/PD-L1 axis together with
$\mathrm{RT}$ is seen as one of the most promising strategy for several solid tumors, and many trials are ongoing with the aim to explore the different possible combinations. Meanwhile, recent experimental findings showed that the interaction between RT and the immune system is far more complex than previously thought, and dose/ fractionation may play a central role for activation, by a mechanism involving the STING pathway and type I interferon release (23). For NSCLC, the combination of immunotherapy and RT might potentially improve local control at the treated site as well as distant control, when a powerful "abscopal" effect is triggered potentiating specific anti-cancer immunity and inducing memory effect (19). RT, especially when combined with immunemodulators, has been shown to broaden the T-cell receptor (TCR) repertoire, achieving maximal tumor rejection (24). The beneficial effects of the combination are expected to be maximal when RT and immune checkpoint inhibitors are used concomitantly or in a close sequence. Moreover, as shown by the clinical results obtained so far $(25,26)$, that will be discussed in details in the next paragraph, RT seems to potentiate the effects of immunotherapy also when given several months before immunotherapy, and this effect was, until now, uniquely observed in NSCLC patients (26). For locally advanced disease, a maintenance approach with anti-PD-1 inhibitors given sequentially seems also to be very effective (25). In these clinical trials, RT was used not as "immunemodulator" but as a radical or palliative treatment at conventional doses. In future studies, as pointed out by De Ruysscher D in a recent commentary (27), it would be possible that RT would be used as a pure trigger of the immune system, opening the possibility to irradiate only partial tumor volumes, or limited metastatic deposits.

\section{Radiotherapy and immunotherapy for locally advanced NSCLC}

The setting of locally advanced NSCLC is remarkably interesting for the combination of immunotherapy and (chemo)-radiotherapy. The main study investigating the efficacy of an anti-PD-1 axis inhibitor in association with radio-chemotherapy for locally advanced NSCLC was the PACIFIC trial (25). This trial compared the PD-L1 inhibitor durvalumab vs. placebo in patients with locally advanced, unresectable stage III NSCLC who did not progress following concurrent platinumbased chemo-radiotherapy. It included 713 patients, who 
were randomized 2:1 to receive durvalumab $10 \mathrm{mg} / \mathrm{kg}$ every 2 weeks or placebo for up to 12 months. The coprimary endpoints were progression-free survival (PFS) and OS. Patients were stratified according to age, sex, and smoking habit (current or former smoker vs. never smoked). Durvalumab was given until disease progression, other therapy initiation, severe collateral effects, or withdrawal of informed consent. The secondary endpoints were duration of response, objective response rate, percentage of patients alive without disease at 12 and 18 months, time to death or distant metastasis, quality of life, pharmacokinetics and immunogenicity. Median PFS resulted significantly higher for patients receiving Durvalumab than for patients receiving placebo (16.8 vs. 5.6 months respectively). The authors pointed out how the gain in PFS was achieved independently of PD-L1 expression $(<25 \%$ vs. $>25 \%)$. The median time to death or distant metastases was 23.2 months for durvalumab $v$ s. 14.6 months for placebo, respectively, the objective response rate was $28.4 \%$ vs. $16 \%$, respectively, and the median duration of response at 12 and 18 months was $72.8 \%$ vs. $56.1 \%$ and $46.8 \%$, respectively. Patients who received durvalumab had a lower incidence of new metastases $(20.4 \%$ vs. $32.1 \%)$ and in particular a lower incidence of brain metastases (5.5\% vs. $11 \%)$. The toxicity of the two arms was comparable, with and incidence of adverse events of any grade of $96.8 \%$ and $94.9 \%$ in patients receiving durvalumab $v s$. placebo, respectively. Grade 3 or 4 adverse events occurred in $29.9 \%$ and $26.1 \%$, with $4.4 \%$ and $5.6 \%$ toxic deaths, respectively.

Pembrolizumab, an anti-PD-1 agent, demonstrated a clear efficacy in advanced NSCLC on phase 1 KEYNOTE 001 trial (28). PD-L1 expression in at least $50 \%$ of tumor cells was selected as cutoff. In a secondary analysis of this trial (26), the authors evaluated all patients enrolled and selected those who received radiotherapy at any timepoint before the administration of the first pembrolizumab cycle. The main endpoint of the study was the evaluation of the impact of previous radiotherapy on PFS and OS, in comparison with patients who did not receive radiotherapy. As secondary endpoint, the toxicity profile of the combination was also evaluated. The status and expression of PD-L1 were determined and patients with a membranous PD-L1 staining at least $1 \%$ were considered positive. Pembrolizumab administration schedule was 2 or $10 \mathrm{mg} / \mathrm{kg}$ every 3 weeks or $10 \mathrm{mg} / \mathrm{kg}$ every 2 weeks until progression of disease, important toxicity or withdrawal of informed consent. Radiotherapy was delivered on thoracic and extra-thoracic sites, mostly as palliative treatment. Of the 97 patients analyzed, 42 previously received any RT, 38 extracranial and 24 thoracic RT (43\%, 39\% and 25\% respectively) with a median time of administration of 9.5 months prior to pembrolizumab. With a median follow-up of 32.5 months for surviving patients, there were 31 progressions in the group of patients undergoing previous radiotherapy and 49 in the group of patients who did not receive it. The combination of radiotherapy and pembrolizumab obtained a significantly longer PFS (4.4 and 2.1 months respectively). Patients who received prior extracranial radiation had a better PFS (6.3 and 2.0 months, respectively). Patients treated with $\mathrm{RT}$ also had better OS: the median survival of the first group was $10.5 \mathrm{vs}$. 5.3 months, and 6-month OS was $73 \%$ vs. $45 \%$, respectively. Again, patients who received a previous extracranial RT had a better OS (11.6 vs. 5.3 months, respectively). Forty-four of 97 patients had grade 3 or greater pulmonary toxicity, without difference among RT vs. non-RT patients. A separate analysis showed that, as far as the side effects were closely related to treatment, 3 patients $(13 \%)$ of the group who received prior RT compared to $1 \%$ of patients who did not receive it developed pulmonary toxicity (17). The combined results of these two trials were favorable in terms of the potential implementation of a chemo-radiotherapy plus immunotherapy approach with anti PD-L1 and PD-1 inhibitors for both locally advanced and stage IV NSCLC. These results are in line with most of the preclinical evidence suggesting that there is window of opportunity for improving tumor control when using radiation and differential immunotherapy strategies. However, the exact mechanism underlying these findings in NSCLC is still unknown, and many clinical questions remain to be answered before a wide application in clinical practice, especially the right group of patients, exact sequence of RTIT, PD-L1 expression levels.

Tecemotide (L-BLP25) was conceived as an antitumor vaccine inducing a specific immune response against MUC-1, a glycoprotein overexpressed and unnaturally glycosylated in many tumors, including NSCLC (29,30). The aberrant protein stimulates cell proliferation, modifies cell adhesion, and promotes metastases. Tecemotide, once internalized by antigen presenting cells (APCs), is able to promote a $\mathrm{T}$ cellmediated response against MUC-1 expressing tumors (31). In the START study, a randomized, international doubleblind phase III trial, the authors enrolled 1,513 NSCLC 
patients with inoperable stage III NSCLC, in objective clinical response or stable disease after definitive chemoradiation. Patients were randomized to receive adjuvant tecemotide for 8 weekly cycles or placebo at a ratio of $2: 1$. OS was not significantly different between the two groups (25.6 vs. 22.3 months, respectively) even if, in a secondary analysis, patients who underwent concomitant chemo-radiotherapy had a better OS (30.8 vs. 20.6 months, respectively) (32). The INSPIRE study aimed at investigating the efficacy of tecemotide associated with best supportive care (BSC) as an alternative to placebo plus BSC after chemo-radiotherapy in stage III NSCLC East-Asian patients. Patients were randomized to receive 8 weekly doses of tecemotide plus maintenance for further 6 weeks, or placebo. The main endpoint of the study was OS, but unfortunately the study was prematurely closed (33). To our knowledge, at the time of writing no other reports were available on the combination of immunotherapy and radio (chemo) therapy for stage III NSCLC.

\section{Ongoing studies and future perspectives}

Radiation therapy for locally advanced NSCLC has evolved over several decades with the aim of developing specific protocols optimized to maximize local tumor control through radiation dose escalation, or the combination with chemotherapy. Many attempts in prolonging OS did fail, and at the same time standard chemo-radiation (platinum doublets and 60 Gy/6 weeks thoracic RT) reached unprecedented results in terms of median OS time in recent randomized comparisons, probably as a consequence of the better initial staging through the wide use of PET-CT, lower toxicity of RT through the use of advanced planning techniques, better quality of life, better supportive care. Despite these positive findings, still most of the patients relapse and die due to disease progression. The growing enthusiasm for immune-oncology and its possible applications in radiation oncology led to a tremendous expansion of preclinical and clinical studies testing various combinations of immunotherapeutic agents and radiation. We summarized the results obtained so far by combining chemo-radiation and immune checkpoint inhibitors in pivotal trials that explored this strategy for stage III NSCLC, with truly encouraging results. However, given the complexity of the field and the paucity of data, we still need to wait for other experimental findings prior to confirm the efficacy of this approach. Particularly, we need to revisit our current understanding of the radiation/immune system interactions in order to develop more tailored strategies in terms of dose/fractionation, timing, target volumes and drugs choice, in light of the recent advances in knowledge coming from translational studies.

In Table 1 we present the ongoing studies retrieved by Clinicaltrials.gov, November 2017. As we can see from the Table, many different options are under investigation by several researchers across USA and Europe. In almost all trials, the target is the PD-1/PD-L1 axis, with the exception of a combination of anti-CTLA-4 and anti-

Table 1 Ongoing trials integrating immunotherapy in the therapeutic management of locally advanced NSCLC

\begin{tabular}{|c|c|c|c|c|c|}
\hline Study & Number & Phase & Immunotherapy & Institution & $\begin{array}{l}\text { Estimated } \\
\text { completion date }\end{array}$ \\
\hline $\begin{array}{l}\text { Cisplatin and etoposide plus radiation } \\
\text { followed by nivolumab/placebo for locally } \\
\text { advanced NSCLC (RTOG 3505) }\end{array}$ & NCT02768558 & III & Anti-PD-1, adjuvant & $\begin{array}{l}\text { RTOG } \\
\text { Foundation }\end{array}$ & October 2024 \\
\hline $\begin{array}{l}\text { Nivolumab combination with standard } \\
\text { first-line chemotherapy and radiotherapy } \\
\text { in locally advanced stage IIIA/B non-small } \\
\text { cell lung carcinoma (NICOLAS) }\end{array}$ & NCT02434081 & ॥ & $\begin{array}{l}\text { Anti-PD-1, } \\
\text { concurrent }\end{array}$ & $\begin{array}{l}\text { European } \\
\text { Thoracic } \\
\text { Oncology } \\
\text { Platform }\end{array}$ & August 2020 \\
\hline $\begin{array}{l}\text { Pembrolizumab in combination with } \\
\text { radiotherapy in locally advanced non-small } \\
\text { cell lung cancer (NSCLC) (PARIS) }\end{array}$ & NCT03245177 & 1 & $\begin{array}{l}\text { Anti-PD-1, } \\
\text { concurrent and } \\
\text { adjuvant }\end{array}$ & $\begin{array}{l}\text { The Christie } \\
\text { NHS } \\
\text { Foundation } \\
\text { Trust }\end{array}$ & November 2020 \\
\hline
\end{tabular}

Table 1 (continued) 
Table 1 (continued)

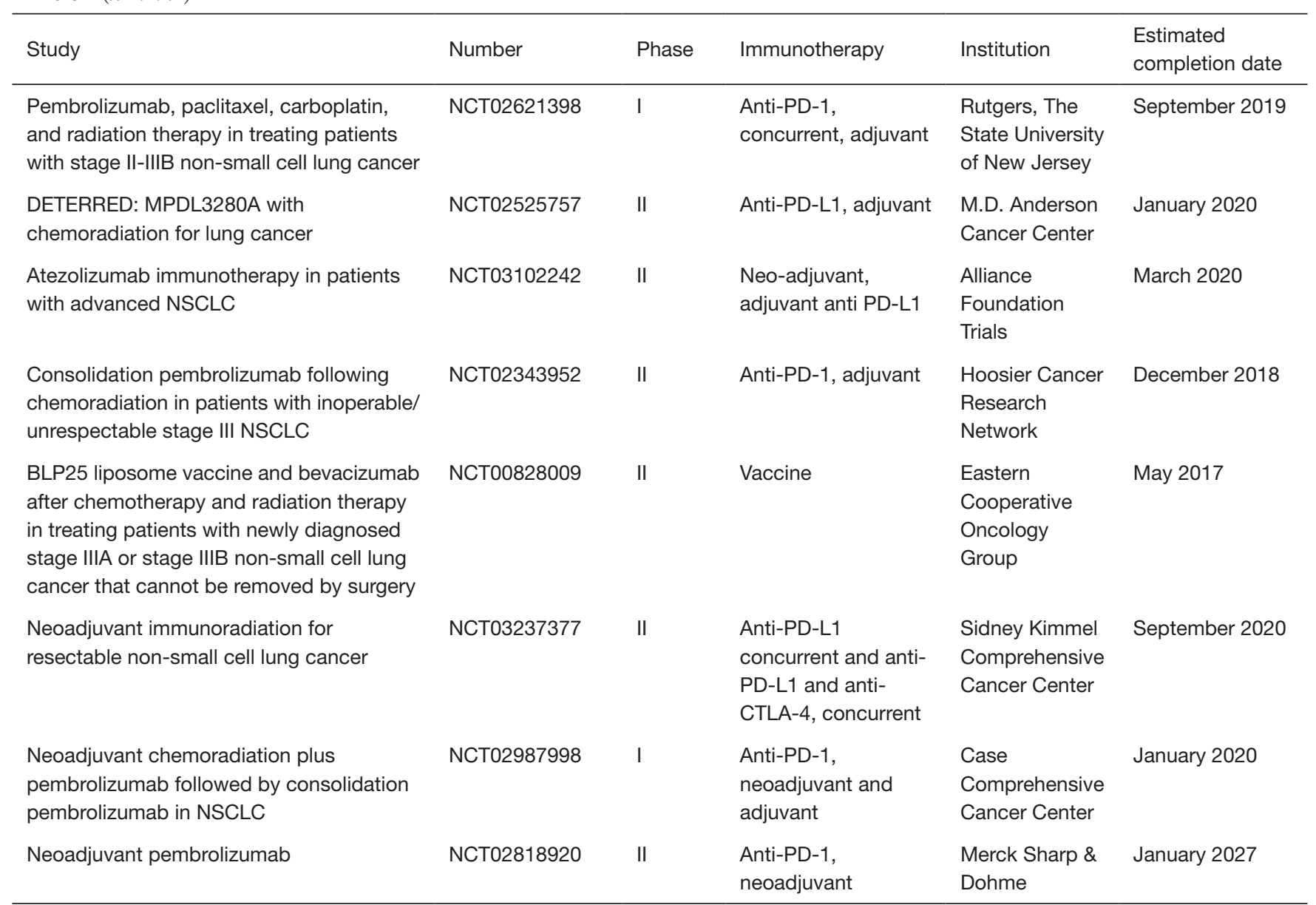

NSCLC, non-small cell lung cancer; RTOG, Radiation Therapy Oncology Group; PD-1, programmed death 1; PD-L1, programmed deathligand 1; CTLA-4, cytotoxic T-lymphocyte-associated protein 4; NHS, National Health System.

PD-L1 agents. Multiple sequences are being tested: a predominance for the consolidation/adjuvant/maintenance setting is evident, however with many studies also integrating immunotherapy at the beginning of chemo-radiation. The latter schedule should be one of the most efficient way to harness at maximum the synergistic effects of chemo-radiation and immunotherapy in terms of boosting the immunestimulating effects, particularly when using anti-PD-L1 agents, given that PD-L1 enhanced expression during RT may be one of the main causes of radioresistance. A particular attention should also be paid to those trials introducing antiPD-1 agents before chemo-radiation, as neo-adjuvant: this innovative approach could be promising, by integrating radiochemotherapy in a tumor micro-environment already modified by immunomodulators, and with a subsequent consolidation phase. When using anti-PD-L1 agents in this setting, PD-
L1 expression levels would be probably necessary to stratify patients.

\section{Acknowledgements}

None.

\section{Footnote}

Conflicts of Interest: The authors have no conflicts of interest to declare.

\section{References}

1. Chen VW, Ruiz BA, Hsieh MC, et al. Analysis of stage and clinical/prognostic factors for lung cancer from SEER 
registries: AJCC staging and collaborative stage data collection system. Cancer 2014;120:3781-92.

2. Rami-Porta R, Asamura H, Travis WD, et al. Lung cancer-major changes in the American Joint Committee on Cancer eighth edition cancer staging manual. CA Cancer J Clin 2017;67:138-55.

3. Aupérin A, Le Péchoux C, Rolland E, et al. Meta-analysis of concomitant versus sequential radiochemotherapy in locally advanced non-small-cell lung cancer. J Clin Oncol 2010;28:2181-90.

4. Bradley JD, Paulus R, Komaki R, et al. Standard-dose versus high-dose conformal radiotherapy with concurrent and consolidation carboplatin plus paclitaxel with or without cetuximab for patients with stage IIIA or IIIB non-smallcell lung cancer (RTOG 0617): a randomised, two-by-two factorial phase 3 study. Lancet Oncol 2015;16:187-99.

5. Feddock J, Arnold SM, Shelton BJ, et al. Stereotactic body radiation therapy can be used safely to boost residual disease in locally advanced non-small cell lung cancer: a prospective study. Int J Radiat Oncol Biol Phys 2013;85:1325-31.

6. Higgins KA, Pillai RN, Chen Z, et al. Concomitant chemotherapy and radiotherapy with SBRT boost for unresectable, Stage III Non-small Cell Lung Cancer: A phase I Study. J Thorac Oncol 2017;12:1687-95.

7. Socinski MA, Stinchcombe TE, Moore DT, et al. Incorporating bevacizumab and erlotinib in the combinedmodality treatment of stage III non-small-cell lung cancer: results of a phase I/II trial. J Clin Oncol 2012;30:3953-9.

8. Borghaei H, Paz-Ares L, Horn L, et al. Nivolumab versus docetaxel in advanced nonsquamous non-small-cell lung cancer. N Engl J Med 2015;373:1627-39.

9. Carbone DP, Reck M, Paz-Ares L, et al. CheckMate 026 Investigators. First-line Nivolumab in stage IV or recurrent Non-Small-Cell Lung Cancer. N Engl J Med 2017;376:2415-26.

10. Herbst RS, Baas P, Kim DW, et al. Pembrolizumab versus docetaxel for previously treated, PD-L1-positive, advanced non-small-cell lung cancer (KEYNOTE-010): a randomised controlled trial. Lancet 2016;387:1540-50.

11. Reck M, Rodríguez-Abreu D, Robinson AG, et al. KEYNOTE-024 Investigators. Pembrolizumab versus chemotherapy for PD-L1-positive non-small-cell lung cancer. N Engl J Med 2016;375:1823-33.

12. Sharabi AB, Lim M, DeWeese TL, et al. Radiation and checkpoint blockade immunotherapy: radiosensitisation and potential mechanisms of synergy. Lancet Oncol 2015;16:e498-509.
13. Galluzzi L, Zitvogel L, Kroemer G. Immunological mechanisms underneath the efficacy of cancer therapy. Cancer Immunol Res 2016;4:895-902.

14. Demaria S, Golden EB, Formenti SC. Role of local radiation therapy in cancer immunotherapy. JAMA Oncol 2015;1:1325-32.

15. Walshaw RC, Honeychurch J, Illidge TM. Stereotactic ablative radiotherapy and immunotherapy combinations: turning the future into systemic therapy? Br J Radiol 2016;89:20160472.

16. Reynders K, Illidge T, Siva S, et al. The abscopal effect of local radiotherapy: using immunotherapy to make a rare event clinically relevant. Cancer Treat Rev 2015;41:503-10.

17. Wang X, Schoenhals JE, Li A, et al. Suppression of type I IFN signaling in tumors mediates resistance to anti-PD-1 treatment that can be overcome by radiotherapy. Cancer Res 2017;77:839-50.

18. Twyman-Saint Victor C, Rech AJ, Maity A, et al. Radiation and dual checkpoint blockade activate non-redundant immune mechanisms in cancer. Nature 2015;520:373-7.

19. Formenti SC, Demaria S. Combining RT and cancer immunotherapy: a paradigm shift. J Natl Cancer Inst 2013;105:256-65.

20. Ngiow SF, McArthur GA, Smyth MJ. Radiotherapy complements immune checkpoint blockade. Cancer Cell 2015;27:437-8.

21. Filippi AR, Fava $P$, Badellino $S$, et al. Radiotherapy and immune checkpoints inhibitors for advanced melanoma. Radiother Oncol 2016;120:1-12.

22. Gong $X, \operatorname{Li} X$, Jiang $T$, et al. Combined radiotherapy and anti-PD-L1 antibody synergistically enhances antitumor effect in non-small cell lung cancer. J Thorac Oncol 2017;12:1085-97.

23. Vanpouille-Box C, Formenti SC, Demaria S. Towards precision radiotherapy for use with immune checkpoint blockers. Clin Canc Res 2017. [Epub ahead of print].

24. Rudqvist NP, Pilones KA, Lhullier C, et al. Radiotherapy and anti-CTLA-4 blockade shape the TCR repertoire of tumor infiltrating T cells. Cancer Immunol Res 2018;6:139-50.

25. Antonia SJ, Villegas A, Daniel D, et al. Durvalumab after chemoradiotherapy in stage iii non-small-cell lung cancer. N Engl J Med 2017;377:1919-29.

26. Shaverdian N, Lisberg AE, Bornazyan K, et al. Previous radiotherapy and the clinical activity and toxicity of pembrolizumab in the treatment of non-small-cell lung cancer: a secondary analysis of the KEYNOTE-001 phase 1 trial. Lancet Oncol 2017;18:895-903. 
27. De Ruysscher D. Radiotherapy and PD-L1 inhibition in metastatic NSCLC. Lancet Oncol 2017;18:840-2.

28. Garon EB, Rizvi NA, Hui R, et al. Pembrolizumab for the treatment of non-small cell lung cancer. N Engl J Med 2015;372:2018-28.

29. Bafna S, Kaur S, Batra SK. Membrane-bound mucins: the mechanistic basis for alterations in the growth and survival of cancer cells. Oncogene 2010;29:2893-904.

30. Raina D, Kosugi M, Ahmad R, et al. Dependence on the MUC1-C oncoprotein in non-small cell lung cancer cells. Mol Cancer Ther 2011;10:806-16.

31. Sangha R, Butts C. L-BLP25: a peptide vaccine

Cite this article as: Filippi AR, Di Muzio J, Badellino S, Mantovani C, Ricardi U. Locally-advanced non-small cell lung cancer: shall immunotherapy be a new chance? J Thorac Dis 2018;10(Suppl 13):S1461-S1467. doi: 10.21037/jtd.2017.12.53 strategy in non-small cell lung cancer. Clin Cancer Res 2007;13:s4652-4.

32. Butts C, Socinski MA, Mitchell PL, et al. START trial team. Tecemotide (L-BLP25) versus placebo after chemoradiotherapy for stage III non-small-cell lung cancer (START): a randomised, double-blind, phase 3 trial. Lancet Oncol 2014;15:59-68.

33. Wu YL, Park K, Soo RA, et al. INSPIRE: A phase III study of the BLP25 liposome vaccine (L-BLP25) in Asian patients with unresectable stage III non-small cell lung cancer. BMC Cancer 2011;11:430. 